\title{
Corrigendum
}

\section{Revenue management in the car rental industry: A stochastic programming approach}

\author{
Alwin Haensel, Michael Mederer and Henning Schmidt \\ Department of Mathematics, VU University Amsterdam, Amsterdam, the Netherlands \\ Journal of Revenue and Pricing Management (2011) 10, 290. doi:10.1057/rpm.2011.2
}

Correction to: Journal of Revenue and Pricing Management advance online publication,

23 April 2010; doi:10.1057/rpm.2010.52

Correspondence: Alwin Haensel, Department of Mathematics, VU University Amsterdam, Amsterdam HV 1081, the Netherlands

E-mail: ahaensel@few.vu.nl
After publication online an error was found in Table 10 of the above article. The heading of the last column should read milliseconds but was incorrectly given as minutes. A corrected version of the table is reproduced below.

Table 10: Computation times and problem sizes

\begin{tabular}{lcccccr}
\hline & Period & Cont. var. & Int. var. & Bin. var. & Constraints & Comp. time (ms) \\
\hline DIP & 1 week & - & 105 & - & 147 & 125 \\
SIP & 1 week & 15708 & 7959 & 7854 & 39417 & 3907 \\
DIP & 2 weeks & - & 210 & - & 294 & 187 \\
SIP & 2 weeks & 32592 & 16506 & 16296 & 81775 & 11750 \\
\hline
\end{tabular}

